\title{
Static correlations in the ordered colloidal systems
}

\author{
O.I.Gerasimov ${ }^{1}$, P.P.J.-M.Schram ${ }^{2}$ \\ 1 Odesa State Hydrometheorological Institute, Odesa, Ukraine \\ 2 Eindhoven University of Technology, Eindhoven, Netherlands
}

Received December 29, 1998

\begin{abstract}
The macroion-macroion correlation function and structure factor are studied within the Ornstein-Zernike $(\mathrm{OZ})$ integral equation closed by hypernetted-chain approximation using the method of critical modes. Developing the results which have recently been received in [1-3] for a case of a model system consisting of a mixture of charged hard spheres, the role of detalization in describing the compound characters is investigated. In particular, it is shown that adding the uncharged particles to the model of the solution of macroparticles and counterions significantly affects the structural parameter of ordering extracted within the critical modes method providing close theoretical and relating experimental results.
\end{abstract}

Key words: correlations, structure factor, suspensions

PACS: $61.20 . G y, 82.40 .-g$

\section{Introduction}

A colloidal suspension is a dispersion of microscopic particles in a liquid. Particles can be regarded as colloids if they are first of all significantly larger than solvent molecules and secondly, if they have a typical linear dimension $R$ small enough to show a significant Brownian motion, i.e., approximately $1 \mathrm{~nm}<R<1 \mu \mathrm{m}$. The investigation of model colloids (particles with well defined size, shape and interactions) in recent decades has proved to be a source of intriguing phenomena, crystallisation of colloidal component into Wigner lattice being an example. The equilibrium thermodynamic properties of an assembly of colloids are formally the same as those of an assembly of atoms with an inter-atomic potential of the same form. Statistical mechanics combined with theories developed for simple liquids and solids, can then be used to calculate the phase behaviour and structure of colloidal suspensions. Thus, in some sense, a suspension of colloidal particles can be regarded as an assembly of s.c. super-atoms. For example, for a suspension of equal-sized (monodispersive) spherical colloids, under suitable conditions we may find the existence of colloidal 
gas, liquid (fluids including) or crystal phases, similar to a simple atomic substance such as argon (for a general reference see [4,5]). We shall pay most attention to the all-round charge stabilised colloidal suspensions. Note, charged colloidal particles can be regarded as macroions. On their surfaces there are ionisable groups, at least some of which dissociate when the particles are dispersed in a polar liquid such as water. The particles themselves acquire a charge $q_{0}$, typically $10^{2}$ to $10^{5}$ elementary charges $e$. The counterions discharged into the liquid move away from the macroion in Brownian motion, but nevertheless they remain in its field of force. This results in an electric double layer surrounding the particle, composed of the counterions and the ions of any electrolyte present in the suspension. When two macroions approach each other, the overlap of their double layers causes a repulsive force which can stabilize the particles against aggregation. At present, it is experimentally proved that ionic colloidal suspensions display the extraordinary structures, in particle distribution, such as gas-like, liquid-like and solid-like (crystal-like and amorphous solid-like or glass-like) distributions [5]. The suspensions showing the crystal-like structures are ideal systems for model studies of metals, since the colloidal structures are analyzed using the optical techniques and their forces are readily manipulated by controlling the composition of the suspension. Furthermore, phase transition phenomena such as crystallization and melting occur sharply. A study of the extraordinary structures of colloidal particles is also helpful in understanding fundamental properties of the state of the substance and electrostatic interactions of macro-ionic system. Of course, both of the two essentially important factors in the characteristic properties for colloidal systems (i.e. an electrostatic interparticle repulsion and an expanded electrical double layers around the particle in the deionized state) are quite different from the features of real metals. Observation of the effect of ordering in colloidal systems is possible even with the naked eye of the iridescent colours and single crystals (or crystallites). Colloidal crystals are surrounded by grain boundaries and are quite similar to the morphology of metals. Iridescent colours of the crystal-like suspension are ascribed to the Bragg diffraction of visible light by the arrayed lattice planes. Lattice spacing of the colloidal crystals is by several thousand times longer compared with that of metals being in the range of light wavelengths. As it was mentioned above, ionic groups on the colloidal surfaces leave their counterions in the suspension, and these excess charges accumulate near the surfaces forming an electrical double layer. The double layer consists of two regions; an inner region composed of adsorbed counterions and a diffusive region containing the remainder of the excess counterions. The counterions in the diffuse region are distributed according to a balance between their thermal motion and the force of electrical attraction with the colloidal spheres. The thickness of the diffuse double layer is approximated by the Debye-screening length $\lambda_{\mathrm{DH}}$,

$$
\lambda_{\mathrm{DH}}=\left(\frac{4 \pi e^{2} n}{\varepsilon k_{\mathrm{B}} T}\right)^{-\frac{1}{2}}
$$

where $e$ is the electronic charge, $\varepsilon$ is the dielectric constant of the solvent, $k_{\mathrm{B}}$ is the Boltzman constant and $n$ is the concentration of the free state cations (and anions) in suspension. 
According to the effective hard sphere model for the deionized colloidal suspension, crystal like ordering is formed when the effective diameter $d_{\text {eff }}$ of the spheres containing the Debye-screening length is close to or larger than average intersphere distance $l$, i.e.

$$
d+2 \lambda_{\mathrm{DH}}>l
$$

where $d$ is the diameter of the sphere.

Introducing the energy parameter $\frac{k_{\mathrm{B}} T}{V}$, and ionic parameter $l$ :

$$
\frac{k_{\mathrm{B}} T}{V}=\frac{k_{\mathrm{B}} T}{\frac{\left(Z_{\mathrm{eff}}\right)^{2}}{\varepsilon l} \exp \left(-Z_{\mathrm{eff}}\right)}, \quad \lambda=\frac{l}{\lambda_{\mathrm{DH}}}
$$

where $Z_{\text {eff }}$ is the effective charge number. One can plot $k_{\mathrm{B}} T / V$ which gives rise to the impression regarding the phase diagram between crystal-like and liquid-like structures. The mentioned typical phase diagram plotted for colloidal silica spheres with a diameter $d=109 \mathrm{~nm}, s: \frac{s}{d}=0.028$ is shown on figure 1 data from [6].

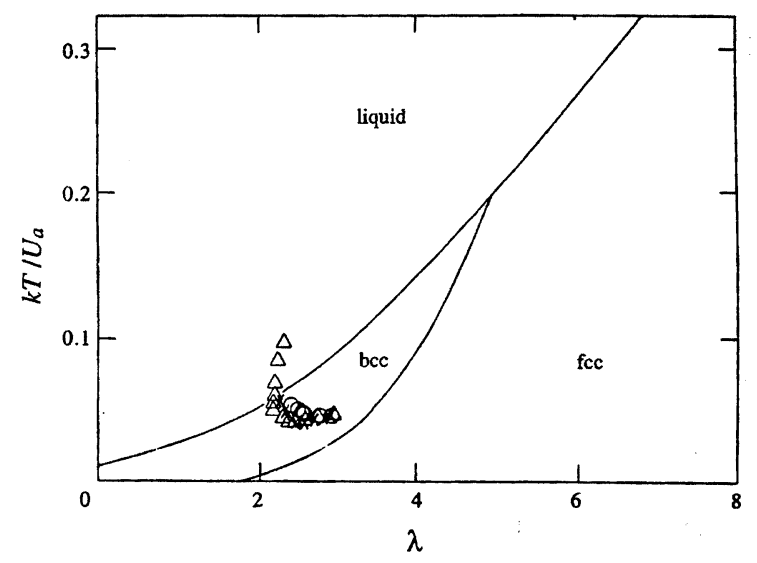

Figure 1. The typical phase diagram between crystal-like and liquid-like structures.

The phase behaviour and structure of suspensions of charge-stabilised colloids has been reviewed in many papers, see for example [4-6]. The common conclusion is the following. At low electrolyte concentrations (deionised suspensions) the double layers can be extensive. The energy of electrostatic repulsion between two particles can then be comparable to the thermal energy $k_{\mathrm{B}} T$ for interparticle spacing of ten or more particle diameters. Such a suspension shows both marked fluid-like ordering and freezing transition at volume fractions $\Phi=\frac{\pi}{6} d^{3} n$ as low as $10^{-4}$. The structure of the very dilute crystals can be either body-centred cubic (bcc) or face-centred cubic (fcc) and frequently near perfect single structures are observed.

\section{Short review of the problem}

Interactions between charged macroparticles in an aqueous environment play an important role in many physical systems (colloidal suspensions, colloid-polymer 
mixtures, solutions of proteins and organic ions in solutions of polymers, solutions of charged micelles and viruses, dusted plasma, etc.) $[4,5,7-11]$. In the theory of colloidal solutions, there is a well-known controversy concerning the neglect of certain interactions in the solution [12-20]. It seems self-evident that the complete statistical mechanical theory contains all interactions between all species in the solutions, macroions, counterions and coins, and solvent, and hence all correlation functions between all species must be considered. Any approximation to this complete theory must necessarily replace true potential energies by potentials of a mean force. The most successful theories of electrolyte solutions are those based on the OrnsteinZernike (OZ) equation [21] with appropriate closure relations [22]. The hypernettedchain (HNC) closure is regarded as the best closure relation for ionic systems such as electrolyte solutions over a wide range of thermodynamic state points, molten salts, and one component plasma. The hypernetted-chain approximation has got the advantage of its accounting for the size of ions directly and of its being suitable for treating the highly asymmetric electrolytes. One of the earliest analysis of the crystallisation phenomena in the macroionic solutions was made by Kirkwood and Hastings [24]. The theory [24] was based on the model of aqueous solution of macroions in the presence of both the counterions and the added salt. It was shown that the theory exhibits a mean field phase transition which was interpreted as a transition to the crystalline phase. The lower bound on the macroion charge necessary for crystallisation was estimated. There are some recent studies of the macroion mixtures based on the HNC integral equation. In [25] the OZ equation was solved in the HNC approximation for several mixtures of electrolytes and uncharged hard spheres. The mixtures that were studied range from a primitive model of simple restricts plus hard spheres of the same size to highly asymmetric electrolytes plus hard spheres of different sizes. The radial distribution functions and thermodynamic properties were monitored. It was found that the presence of neutral particles changes the nature of the interaction between the charged particles. However, no indications of a phase transition was found for any cases analyzed in this work. Adopting the same technique V.Vlachy [26] presented the radial distribution functions for systems containing mono- or divalent counterions for a range of macroion concentrations. A qualitative and a good enough coincidence of the results obtained in the above mentioned works $[25,26]$ is observed. However, both works ignore the molecular nature of the solvent. D.Duh and A.Haymet [13] applied the HNC approximation to reanalyze the small angle neutron scattering (SANS) data of Kunz et al [27] for bulky ions in solution. The pair correlation function was obtained from the potential model via the HNC approximation. The partial structure factor and the coherent intensity of neutrons scattered elastically from an ionic solution was related, as usual, to the Fourier transform of the pair correlation function. By exhibiting an alternate set of fitting parameters they have shown conclusively that fitting SANS data with a model potential may not be unique even within a given integral equation approximation. Hence the solvent-averaged pair correlation function obtained using this method does not necessarily represent the real structure of the electrolyte and thus the conclusions drawn from the fitted pair correlation functions, rather than di- 
rectly from the experimental data, are inconclusive. The authors pointed out that in colloidal solutions, the treatment of the solvent as a continuum may be possibly justified by the fact that the diameter of colloidal particle is usually orders of magnitude larger than that of the solvent molecule, and hence the solvent contribution to the structure factor will appear in a different (high) range of the wave-vector values. However, for example the studies [28] seem to cost doubt even in this case, hence the justification of the above approximation is open. Besides the problem of justification of the solvent averaged model, in the integral equation theory of ionic solutions a problem arises of the validity of the HNC approximation by itself $[22,29-$ 31]. It is known that, for the short-ranged repulsive forces the Percus-Yevick (PY) approximation works effectively, despite the fact that it includes fewer diagrams in a pair correlation function and in a direct correlation function. The HNC closure has been found to be effective for systems with long-range forces. However, for model electrolytes in the strong coupling and in the low concentration regime, where the charge association occurs, the HNC approximation begins to break down and yields unphysical predictions for the structure and critical behaviour, apparently because the HNC cannot account for ion association [32]. Presumably, the short-range forces which are better handled by the PY closure become more important, resulting in the breakdown of the HNC approximation. There are at least two routs for improving the HNC approximation. One is the direct inclusion of association effects addressed in [29]. The other is adding the bridgefunction contribution to the cluster expansion for the pair correlation function (see for example [29] and references therein), although ion association is not addressed explicitly. A.Vompe and G.Martynov [31] proposed self-consistent solutions to the Ornstein-Zernike equation where the approximate closure is completely replaced by a bridge function expansion, whose main advantage is the improvement of correlation functions. Unknown coefficients of this expansion were found from the principle of total thermodynamic consistency. The used form of expansion performed a fast convergence and presented an efficient and universal solution of closure problem that is of special importance for a problem of phase transitions [24]. Subsequently, in our further consideration we will adopt the HNC closure. We hope that the HNC closure should be valid for a number of experimentally studied colloidal suspensions [4-8], since the distances between colloidal particles are usually orders of magnitude some times larger than the diameter of colloidal particle.

\section{Models and methods}

Let us consider a water suspension of polystyrene colloidal particles with a size of order of $a \approx 0.1 \mu \mathrm{m}$. We note that in recent experiments [4-8] the crystalline phase formed by colloidal particles with the lattice constant $R_{0} \approx 1 \mu \mathrm{m}$ could be generated. Hereinafter we restrict our consideration by the three main cases. At first we shall suppose that the system under consideration can be described as a onecomponent plasma of finite size ions, the counterions and solvent molecules being treated as a uniform neutralizing background which determines the screening in the 
system. Secondly we shall consider a two component model (colloidal particles plus counterions), where the solvent is treated as a continuum, and specific ion-solvent interactions are neglected. Finally, all interactions between all species in the solution, macroions, counterions and solvent (water) molecules are present explicitly in our calculation. The HNC approximation belongs to a family of integral equations which are rooted in the OZ equation. The OZ equation is a general relationship between the static correlation function $G^{\sigma \sigma^{\prime}}\left(\vec{R}_{1}, \vec{R}_{2}\right)=F_{2}^{\sigma \sigma^{\prime}}\left(\vec{R}_{1}, \vec{R}_{2}\right)-1$, where $F_{2}^{\sigma \sigma^{\prime}}\left(\vec{R}_{1}, \vec{R}_{2}\right)$ is the binary distribution function, and the direct $G_{2}^{\sigma \sigma^{\prime}}\left(\vec{R}_{1}, \vec{R}_{2}\right)$ correlation function. For a mixture of $r$ species the $\mathrm{OZ}$ equation is

$$
G^{\sigma \sigma^{\prime}}\left(\vec{R}_{1}, \vec{R}_{2}\right)=G_{2}^{\sigma \sigma^{\prime}}\left(\vec{R}_{1}, \vec{R}_{2}\right)
$$

where $n_{\sigma}$ is the number density of $\sigma$-species and the sum of integrals represent the indirect contribution to the static correlation function. The superscript $\sigma$ denotes neutral solvent molecules $(s=1)$, microions $(s=2)$ and colloidal particles $(s=3)$.

Following [1-3], note that in the equilibrium state the problem of calculating the static correlation function is reduced to determining the effective potentials for the particles of species $\sigma$ and $\sigma^{\prime}$ located at the points $\vec{R}_{1}$ and $\vec{R}_{2}$ respectively

$$
F_{2}^{\sigma \sigma^{\prime}}\left(\vec{R}_{1}, \vec{R}_{2}\right)=\exp \left(-\frac{V_{\text {eff }}^{\sigma \sigma^{\prime}}\left(\vec{R}_{1}, \vec{R}_{2}\right)}{T}\right),
$$

where $T$ is the absolute temperature in the energy units. Using the hypernetted chain approximation [17] one can obtain:

$$
\begin{gathered}
V_{\text {eff }}^{\sigma \sigma^{\prime}}\left(\vec{R}_{1}, \vec{R}_{2}\right)=V^{\sigma \sigma^{\prime}}\left(\vec{R}_{1}, \vec{R}_{2}\right)-\sum_{\sigma^{\prime \prime}=1}^{r} n_{\sigma^{\prime \prime}} T \\
\times\left[\exp \left(-\frac{V_{\mathrm{eff}}^{\sigma \sigma^{\prime}}\left(\vec{R}_{1}, \vec{R}_{3}\right)}{T}\right)-1+\frac{1}{T} V_{\mathrm{eff}}^{\sigma \sigma^{\prime}}\left(\vec{R}_{1}, \vec{R}_{3}\right)-\frac{1}{T} V^{\sigma \sigma^{\prime}}\left(\vec{R}_{1}, \vec{R}_{3}\right)\right] \\
\times\left[\exp \left(-\frac{V_{\mathrm{eff}}^{\sigma^{\prime \prime} \sigma^{\prime}}\left(\vec{R}_{3}, \vec{R}_{2}\right)}{T}\right)-1\right],
\end{gathered}
$$

where $V^{\sigma \sigma^{\prime}}(R)$ is the direct pair potential.

We assume that $V^{\sigma \sigma^{\prime}}(R)$ and $V_{\text {eff }}^{\sigma \sigma^{\prime}}(\vec{R})$ can be divided into a universal short-range part $V_{0}^{\sigma \sigma^{\prime}}(\vec{R})$ and long-range part $\nu^{\sigma \sigma^{\prime}}(\vec{R})$ and $\tilde{V}^{\sigma \sigma^{\prime}}(\vec{R})$, respectively, according to

$$
\begin{aligned}
V^{\sigma \sigma^{\prime}}(\vec{R}) & =V_{0}^{\sigma \sigma^{\prime}}(\vec{R})+\nu^{\sigma \sigma^{\prime}}(\vec{R}), \\
V_{\mathrm{eff}}^{\sigma \sigma^{\prime}}(\vec{R}) & =V_{0}^{\sigma \sigma^{\prime}}(\vec{R})+\tilde{V}^{\sigma \sigma^{\prime}}(\vec{R}), \\
V_{0}^{\sigma \sigma^{\prime}}(\vec{R}) & =\left\{\begin{array}{cc}
\infty, & R<a_{\sigma \sigma^{\prime}} \\
0, & R<a_{\sigma \sigma^{\prime}}
\end{array} .\right.
\end{aligned}
$$

where $a_{\sigma \sigma^{\prime}}=a_{\sigma}+a_{\sigma^{\prime}}$, being the radius of the solid core for the particles of species $\sigma, \vec{R}=\vec{R}_{1}+\vec{R}_{2}$. 
Substituting equation (7) into equation (6), one obtains the equation

$$
\begin{gathered}
\tilde{V}^{\sigma \sigma^{\prime}}(\vec{R})=\nu^{\sigma \sigma^{\prime}}(\vec{R})-\sum_{\sigma^{\prime \prime}=1}^{r} n_{\sigma^{\prime \prime}} T \\
\times \int \mathrm{d} \vec{R}^{\prime}\left(\left[\exp \left(-\frac{V^{\sigma \sigma^{\prime}}(\vec{R})}{T}\right)+\operatorname{frac} 1 T \nu^{\sigma \sigma^{\prime}}\left(\vec{R}^{\prime}\right)\right] \Theta\left(R^{\prime}-a_{\sigma \sigma^{\prime}}\right)-1\right) \\
\times\left[\Theta\left(\left|\vec{R}-\vec{R}^{\prime}\right|-a_{\sigma^{\prime \prime} \sigma^{\prime}}\right) \exp \left(-\frac{\tilde{V}^{\sigma \sigma^{\prime}}\left(\vec{R}-\overrightarrow{R^{\prime}}\right)}{T}\right)-1\right]
\end{gathered}
$$

which is valid only at $R>a_{\sigma \sigma^{\prime}}$.

Linearizing equation (9) with respect to $V^{\sigma \sigma^{\prime}} / T$ we find the equation describing the behaviour of the effective potentials far from the critical point

$$
\begin{gathered}
\tilde{V}^{\sigma \sigma^{\prime}}(\vec{R})=\nu^{\sigma \sigma^{\prime}}(\vec{R})-\sum_{\sigma^{\prime \prime}=1}^{r} n_{\sigma^{\prime \prime}} T \\
\times \int \mathrm{d} \vec{R}^{\prime}\left[\Theta\left(a_{\sigma \sigma^{\prime}}-R^{\prime}\right)+\frac{1}{T} \nu^{\sigma \sigma^{\prime}}\left(R^{\prime}\right) \Theta\left(R^{\prime}-a_{\sigma \sigma^{\prime}}\right)\right] \\
\times\left[\Theta\left(a_{\sigma^{\prime \prime} \sigma^{\prime}}-\left|\vec{R}-\vec{R}^{\prime}\right|\right)+\frac{1}{T} \tilde{V}^{\sigma^{\prime \prime} \sigma^{\prime}}\left(\vec{R}-\overrightarrow{R^{\prime}}\right) \Theta\left(\left|\vec{R}-\vec{R}^{\prime}\right|-a_{\sigma^{\prime \prime} \sigma^{\prime}}\right)\right] .
\end{gathered}
$$

Equation (10) can be solved by the Fourier method. The Fourier representation of equation (7) may be written as

$$
\tilde{V}_{k}^{\sigma \sigma^{\prime}}+\sum_{\sigma^{\prime \prime}=1}^{r} n_{\sigma^{\prime \prime}} \Lambda^{\sigma \sigma^{\prime \prime}}(\vec{k}) \tilde{V}_{k}^{\sigma^{\prime \prime} \sigma^{\prime}}=\nu_{k}^{\sigma \sigma^{\prime}}-T \sum_{\sigma^{\prime \prime}=1}^{r} n_{\sigma^{\prime \prime}} \Lambda^{\sigma \sigma^{\prime \prime}}(\vec{k}) \Theta_{k}^{\sigma^{\prime \prime} \sigma^{\prime}},
$$

where

$$
\begin{gathered}
\tilde{V}_{k}^{\sigma \sigma^{\prime}}=\int_{R>a_{\sigma \sigma^{\prime}}} \mathrm{d} \vec{R} \exp (-\mathrm{i} \vec{k} \vec{R}) \tilde{V}^{\sigma \sigma^{\prime}}(\vec{R}), \\
\nu_{k}^{\sigma \sigma^{\prime}}=\int_{R>a_{\sigma \sigma^{\prime}}} \mathrm{d} \vec{R} \exp (-\mathrm{i} \vec{k} \vec{R}) \nu^{\sigma \sigma^{\prime}}(\vec{R}), \\
\Lambda^{\sigma \sigma^{\prime}}(\vec{k})=\Theta_{k}^{\sigma \sigma^{\prime}}+\frac{1}{T} \nu_{k}^{\sigma \sigma^{\prime}}, \\
\Theta_{k}^{\sigma \sigma^{\prime}}=\int_{R>a_{\sigma \sigma^{\prime}}} \mathrm{d} \vec{R} \exp (-\mathrm{i} \vec{k} \vec{R})=\frac{4 \pi}{k^{3}}\left(\sin k a_{\sigma \sigma^{\prime}}-k a_{\sigma \sigma^{\prime}} \cos k a_{\sigma \sigma^{\prime}}\right) .
\end{gathered}
$$

The equation (11) can be formally simply solved.

Static structure factors can be defined by the relation

$$
S^{\sigma \sigma^{\prime}}(\vec{k})=\delta_{\sigma \sigma^{\prime}}+\sqrt{n_{\sigma} n_{\sigma^{\prime}}} \int \mathrm{d} \vec{R} \exp (-\mathrm{i} \vec{k} \vec{R})\left[\exp \left(-\frac{V_{\text {eff }}^{\sigma \sigma^{\prime}}(\vec{R})}{T}\right)-1\right]
$$




\section{One-, two- and three-component model for colloidal suspensions}

One can assume as a model that the structural properties of a colloidal suspension are governed only by a finite ion screened Coulomb interparticle pair potential. It is known that for dilute suspensions the effective macroion interaction potential could be approximately described by the Derjaguin-Landau-Verwey-Overbeek (DLVO) potential (see for example [33]):

$$
\nu(R)=\frac{Z_{\mathrm{eff}}^{2} e^{2}}{\varepsilon R} \exp \left(-\frac{R}{R_{\mathrm{D}}}\right), \quad Z_{\mathrm{eff}}=\frac{Z \exp \left(a / R_{\mathrm{D}}\right)}{1+a / R_{\mathrm{D}}}, \quad R_{\mathrm{D}}^{2}=\frac{T \varepsilon}{4 \pi n_{c} e^{2}},
$$

where $R_{\mathrm{D}}$ - Debye radius, $a \equiv a_{3}$ is the radius of a macroion with charge $Z e(Z \equiv$ $\left.Z_{3}\right), n_{c} \equiv n_{2}$ is the number density of the counterions, is the relative permittivity of water. This potential leads directly to an effective Yukawa pair potential with a rescaled macroion charge $Z_{\text {eff }}$.

The Ornstein-Zernike equation (4) in this case is reduced to those for the one component mixture $(r=1)$. Taking into account notation adopted above, one can obtain the following results

$$
\begin{aligned}
\tilde{V}_{k}^{33} & =\frac{T}{n_{3}}\left(1-n_{3} \Theta_{k}^{33}\right)-\frac{T}{n_{3}} \frac{1}{1+n_{3}\left(\Theta_{k}^{33}+\frac{1}{T} \nu_{k}^{33}\right)}, \\
S_{k}^{33}(\vec{k}) & =\frac{1}{1+n_{3}\left(\Theta_{k}^{33}+\frac{1}{T} \nu_{k}^{33}\right)},
\end{aligned}
$$

where $\nu_{k}^{33}$ is determined by the result of the substitution (14) in (12) :

$$
\nu_{k}^{33}=\frac{4 \pi Z_{\mathrm{eff}}^{2} e^{2}}{\varepsilon k^{2}} \frac{\exp \left(-\frac{a_{33}}{R_{\mathrm{D}}}\right)}{\left(\frac{1}{R_{\mathrm{D}} k}\right)^{2}+1}\left(\frac{1}{R_{\mathrm{D}} k} \sin k a_{33}+\cos k a_{33}\right) .
$$

We will attribute the generation of the s.c. critical modes (singularities) in $S_{k}^{33}(\vec{k})$ to the emerging of instabilities which could lead to structural (phase) transitions. The reciprocal of the wave vector $k_{0}$ when such a singularity occurs, could be treated as an estimate for the structural parameter of ordering for example lattice constant $R_{0}=$ $\frac{2 \pi}{k_{0}}$ of colloidal system [1-3,23,24]. In our case the positions of possible singularities of the structure factor (15) are determined by the equation:

$$
1+n_{3}\left(\Theta_{k}^{33}+\frac{1}{T} \nu_{k}^{33}\right)=0
$$

which under the parameters, valued for the respective experiments $[7,8]$, namely: $N_{3}=3.3 \cdot 10^{12} \mathrm{~cm}^{-3}, a_{3}=5.5 \cdot 10^{-6} \mathrm{~cm}, T=293^{\circ} \mathrm{K}$, have been solved numerically. It was shown that the structure factor $S^{33}(\vec{k})$ has got two types of singularities corresponding the close-packed structure and ordered colloidal (crystal-like) packed structure of the considered model system. As the charge of colloidal particle decreases, we observe at $\left|Z_{3}\right|=170$ the lost of singularities of the structure factor $S^{33}(\vec{k})$ and the coexistence point of the close-packed and crystal-like packed phases. 
The colloidal suspension could be also considered as a mixture of charged hardsphere particles (counterions $-\sigma=2$, and colloidal particles $-\sigma=3$ ) immersed in a continuous dielectric with the relative permittivity $\varepsilon$. The discrete structure of the solvent in this model could be neglected. The long-range part of the potential energy between particles of species $\sigma$ and $\sigma^{\prime}, \nu^{\sigma \sigma^{\prime}}(R)$ is

$$
\nu^{\sigma \sigma^{\prime}}(R)=\frac{Z_{\sigma} Z_{\sigma^{\prime}} e^{2}}{\varepsilon R}, \quad R>a_{\sigma \sigma^{\prime}},
$$

where $\sigma, \sigma^{\prime}=2,3$. In the case of the interparticle potential having the form (18), we derive, using equation (13) with respect to equation (12), the following expression for the structure factor $S^{33}(\vec{k})$ :

$$
S^{33}(\vec{k})=\Pi_{22}(\vec{k}) / \operatorname{det}\left|\Pi_{22}(\vec{k})\right|, \quad \Pi_{22}(\vec{k})=\delta_{\sigma \sigma^{\prime}}+n_{\sigma^{\prime}} \Lambda^{\sigma \sigma^{\prime}}(\vec{k}),
$$

where $\nu_{k}^{\sigma \sigma^{\prime}}$ in equation (12) is taken in the form:

$$
\nu_{k}^{\sigma \sigma^{\prime}}=\frac{4 \pi Z_{\sigma} Z_{\sigma^{\prime}} e^{2}}{\varepsilon} \frac{\cos k a_{\sigma \sigma^{\prime}}}{k^{2}}, \quad \sigma, \sigma^{\prime}=2,3
$$

Equation

$$
\operatorname{det}\left|\Pi_{22}(\vec{k})\right|=0
$$

determines the singularities of the structure factor $S^{33}(\vec{k})$ the emerging of which indicates, accordingly, the critical mode behaviour of the system. It is possible to show that the character of the singularities of the structure factor $S^{33}(\vec{k})$ of the two-component model is qualitatively the same but quantitatively different from the one-component model.

Finally, consider the a model where all the macroion, counterion, and solvent molecule interactions are present explicitly in calculation. The long range part of the potential of interaction between the species $\sigma, \sigma^{\prime}=1,2,3$ is defined by

$$
\begin{aligned}
\nu^{11}(R) & =-\frac{3\left(\overrightarrow{d \vec{l}}_{R}\right)\left(\vec{d}_{m} \vec{l}_{R}\right)-\left(\vec{d} \vec{d}_{m}\right)}{R^{3}}, \\
\nu^{1 \sigma}(R) & =\frac{Z_{\sigma} e\left(\overrightarrow{d \vec{l}}_{R}\right)}{R}, \quad \sigma=2,3 \\
\nu^{\sigma \sigma^{\prime}}(R) & =\frac{Z_{\sigma} Z_{\sigma^{\prime}} e^{2}}{R}, \quad \sigma, \sigma^{\prime}=2,3
\end{aligned}
$$

where $\vec{d}, \vec{d}_{m}\left(|\vec{d}|=\left|\vec{d}_{m}\right|\right)$ are the dipole moments of the two water molecules, $\vec{R}$ is the radius vector which connect the centers of mass of the two water molecules $\sigma=1$ (equation (22)) and the center of mass of the water molecule and particle of species $\sigma=2,3, \vec{l}_{R}$ is the unit vector directed parallel to $\vec{R}$. The structure factor $S^{33}(\vec{k})$, which is of interest to us, in this case takes the form:

$$
S^{33}\left(\vec{k}, \vec{d}, \vec{d}_{m}\right)=\Pi_{33}^{-1}(\vec{k})=\frac{\Pi_{11}(\vec{k}) \Pi_{22}(\vec{k})-\Pi_{12}(\vec{k}) \Pi_{21}(\vec{k})}{\operatorname{det}\left|\Pi_{22}(\vec{k})\right|},
$$


where the elements of the matrix $\Pi_{\sigma \sigma^{\prime}}(\vec{k})$ are determined by the equation (19). The Fourier transformation of equation (22) has the form

$$
\begin{aligned}
\nu_{k}^{1 \sigma} & =-\frac{4 \pi i e Z_{\sigma} \vec{k} \vec{d}}{k^{2}} \frac{\sin k a_{1 \sigma}}{k a_{1 \sigma}}, \quad \sigma=2,3, \\
\nu_{k}^{\sigma i} & =-\nu_{k}^{1 \sigma}, \\
\nu_{k}^{\sigma \sigma^{\prime}} & =\frac{4 \pi Z_{\sigma} Z_{\sigma^{\prime}} e^{2}}{k^{2}} \cos k a_{\sigma \sigma^{\prime}}, \quad \sigma, \sigma^{\prime}=2,3 .
\end{aligned}
$$

One can divide the consideration of the three component model into the two cases. First consider the situation when the dipole moments of the water molecules are strongly correlated to each other $\vec{d}=\vec{d}_{m}$.

Using equation (12) and equation (22) we obtain the Fourier transform of $\nu^{11}(R)$ :

$$
\nu_{k}^{11}=\frac{4 \pi d^{2}}{\left(k a_{11}\right)^{2}}\left(\frac{\sin k a_{11}}{k a_{11}}-\cos k a_{11}\right)\left[3\left(\frac{\vec{k} \vec{d}}{k d}\right)^{2}-1\right],
$$

where $d=|\vec{d}|$.

The average value of the $S^{33}(\vec{k}, \vec{d})$ is determined by the integral:

$$
\left\langle S^{33}(\vec{k}, \vec{d})\right\rangle=\int S^{33}(\vec{k}, \vec{d}) \mathrm{d} \Omega,
$$

where $\mathrm{d} \Omega=\sin \vartheta \mathrm{d} \vartheta \mathrm{d} \varphi$ is the element of the spherical angle (the wave vector $\vec{k}$ is directed along the $Z$-axis). Numerical analysis of equation (26) shows that under the certain values of the wave vector $k$ for the fixed charge number $Z_{3}$, the integrand has singularities over a domain of integration.

In this case we obtain the definitive increase of the estimated value for the lattice parameter that suggests the emerging of the instabilities in the existing phase.

At last, supposing that the dipole moments of the water molecules have got arbitrary chaotic orientations in space, we have the following:

$$
\nu_{k}^{11}=\frac{4 \pi}{\left(k a_{11}\right)^{2}}\left(\frac{\sin k a_{11}}{k a_{11}}-\cos k a_{11}\right)\left[3 \frac{(\vec{k} \vec{d})\left(\vec{k} \vec{d}_{m}\right)}{k^{2}}-\vec{d} \vec{d}_{m}\right]
$$

The respective structure factor $S^{33}\left(\vec{k}, \vec{d}, \vec{d}_{m}\right)$ is given by

$$
\left\langle S^{33}\left(\vec{k}, \vec{d}, \vec{d}_{m}\right)\right\rangle=\frac{1}{(4 \pi)^{3}} \int S^{33}\left(\vec{k}, \vec{d}, \vec{d}_{m}\right) \mathrm{d} \Omega \mathrm{d} \Omega_{m} \mathrm{~d} \Omega_{0}
$$

where $\mathrm{d} \Omega_{i}=\sin \vartheta_{i} \mathrm{~d} \vartheta_{i} \mathrm{~d} \varphi_{i} ; \vartheta, \vartheta_{m}, \vartheta_{0}$ are spherical angles between $\vec{k}$ and $\vec{d}, \vec{k}$ and $\vec{d}_{m}$, $\vec{d}$ and $\vec{d}_{m}$ vectors, respectively. The distribution function of the orientations of the dipole moments in equation (29) is assumed to be equal to unity. Carrying out the integration in equation (29) by the variables $\mathrm{d} \Omega$ and $\mathrm{d} \Omega_{m}$, and making the numerical analysis of the retaining integral with respect to $\vartheta_{0}$, one can obtain the definitive 
values of the wave vector $\vec{k} \equiv \vec{k}_{0}$, under which the structure factor $\left\langle S^{33}\left(\vec{k}, \vec{d}, \vec{d}_{m}\right)\right\rangle$ has got singularities. Adopted model demonstrates qualitatively similar behaviour with respect to the three- (with correlated dipole moments) component model. Quantitative discrepancy lies (see simultaneous representation on figure 1) in the magnitudes of the critical values of the wave vector $\vec{k}_{0}$ under which critical mode generates.

\section{Discussion and conclusions}

In summary, we have solved the HNC equation in linear approximation for the macroionic water solution. The procedure for simulation of the ordering tendency in such a system is proposed and implemented based on the singularities of the structure factor in terms of critical modes approach. Applying this method, the structural properties of the water suspensions of polystyrene colloidal particles are analysed. First we considered the simplest case: one-component plasma of finite size ions with the pair-wise additive DLVO interparticle interaction potential. The model neglects the discrete nature of suspending fluid as well as the counterion degrees of freedom imposed only effectively. We analysed the character of singularities of the structure factor $S^{33}(\vec{k})$ and estimated the parameter of ordering lattice constant of colloidal crystal for the different values of the charge number of colloidal particles. We found that under the experimentally reported values from $[7,8]$ of the charge number $Z_{3}=-360$, the number density $n_{3}=3.3 \cdot 10^{12} \mathrm{~cm}^{-3}$, the temperature $T=293^{\circ} \mathrm{K}$, and the diameter of colloidal particles $a_{33}=1.11 \cdot 10^{-5} \mathrm{~cm}$, the estimated value for the lattice constant of colloidal crystal is $R_{0}=3.73 \cdot 10^{-5} \mathrm{~cm}$, which is approximately 2.7 times smaller than the experimental one $\left(\cong 10^{-4} \mathrm{~cm}\right)$ from $[7,8]$. As the charge number $\left|Z_{3}\right|$ decreases, we observe the effect of the existence of the minimal charge for the critical mode generation method which is well consistent with other theoretical [34] and experimental [4-8] predictions, that are operated by the approaches in describing the order-disorder transitions which are different from the one proposed above. Thereafter, we explore a two component model (colloidal particles plus counterions), where the solvent is treated as a continuum, and specific ion-solvent interactions are neglected. We obtain, that with the above mentioned parameters of colloidal suspension, the estimated value of the lattice constant of the possible colloidal crystal is $R_{0}=4.29 \cdot 10^{-5} \mathrm{~cm}$, which is in a better agreement with the experimental value. As the charge of colloidal particles decreases, the system again exhibits the effect of minimal charge. As the charge number $\left|Z_{3}\right|$ reaches the value $\left|Z_{3}\right|=146$, we observe the loss of singularities in the structure factor $S^{33}(\vec{k})$.

We also conclude that the two-component model leads to a more precise magnitude for the lattice constant of colloidal crystal than the one-component model. However, note that qualitatively DLVO potential works well enough as an initial potential to predict the character of singularities in the structure factor $S^{33}(\vec{k})$.

Finally we explore the most complicated case of a three-component model when all interactions between all species in the solution, macroions, counterions and solvent (water) molecules are present explicitly in calculation. We divided consideration of the three component model into the two cases. First, we considered the 
case when the dipole moments of the water molecules are strongly correlated to each other. Averaging the structure factor $S^{33}(\vec{k}, \vec{d})$, we obtained that, with the accepted values of parameters of colloidal suspension, the lattice constant of the possible colloidal crystal is $R_{0}=5.56 \cdot 10^{-5} \mathrm{~cm}$. The minimum charge number again exists and quails. Finally we consider the case when the dipole moments of the water molecules are noncorrelated. In this case we find the magnitude of the lattice constant $R_{0}=6.73 \cdot 10^{-5} \mathrm{~cm}$, the respective minimal charge $\left|Z_{3}\right|=140$. Here we conclude that the three component model gives a better agreement with the experimental value of the lattice constant $R_{0}$ than it was in the case of one- and two component models.

Authors acknowledge INTAS for financial support of the research.

\section{References}

1. Gerasimov O.I., Schram P.P.J.M., Sitenko A.G., Zagorodny A.G. Critical behaviour of effective potentials in colloidal suspensions within the charged hard-sphere model. // Physica B, 1996, vol. 228, p. 40-51.

2. Gerasimov O.I., Schram P.P.J.M., Sitenko A.G., Zagorodny A.G. Critical behaviour of effective potentials in colloidal suspensions within the charged hard-sphere model. - In: The Workshop on complex fluids and plasmas, Eindhoven univ. of Technology, September, 1995.

3. Gerasimov O.I., Schram P.P.J.M., Kilyan A.N. Kinetic model of ordered colloidal suspension. - In: Thes. of INTAS-Ukraine, Workshop on Condensed Matter Physics, Lviv, May, 1998, p.47.

4. Pieranski P. Colloidal crystals. // Contemp. Phys., 1983, vol. 24, No. 1, p. 25-73.

5. Observation, Prediction and Simulation of Phase Transitions in Complex Fluids (ed. Bans M. et al.), the Netherlands, Kluwer Academic Publishers, 1995.

6. Macro-ion Characterization. From Dilute Solutions to Complex Fluids. (ed. Schmitz K.S.). Washington, DC, American Chemical Society, 1994.

7. Derksen J., Van de Water W. Hydrodynamics of colloidal crystals. // Phys. Rev. A, 1992, vol. 8 , p. 5660 .

8. Hoppenbrouwers M., Van de Water W. Charged colloidal systems. - In: The Workshop on complex fluids and plasmas. Eindhoven univ. technology, September, 1995.

9. Van de Vorst B., Van den Ende D., Mellema J. Dynamic measurements on latex crystals. - In: The Workshop on complex fluids and plasmas. Eindhoven univ.of technology, September, 1995.

10. Monovoukas Y., Gast A.P. The experimental phase diagram of charged colloidal suspensions. // J. Coll. and Interface Sci., 1989, vol. 128, p. 2.

11. Hu Y., Glass J.L., Griffith A.E. Observation and simulation of electrohydrodynamic instabilities in aqueous colloidal suspensions. // J. Chem. Phys., 1994, vol. 100, p. 4674.

12. Henderson D., Lozada-Cassou M. Classical colloidal liquids. // J. Colloid Interface Sci., 1986, vol. 114, p. 180.

13. Duh D., Haymet D. Integral equation theory for charged liquids: The structure of macroionics in solution and the inversion of experimental data // J. Chem. Phys., 1994, No. 3, p. 100. 
14. Hayter J.B., Penfold J. An analytic structure factor for macroionics solutions. // Mol. Phys., 1981, vol. 42, No. 1, p. 109.

15. Hansen J.P., Hayter J.B. A rescaled MSA structure factor for dilute charged colloidal dispersions. // Mol. Phys., 1982, vol. 46, No. 3, p. 651.

16. Löwen H. Charged rodlike colloidal suspensions: An ab initio approach. // J. Chem. Phys., 1994, vol. 100, No. 9.

17. Löwen H. Interparticle interactions and crystallization transition in charged colloidal suspensions. - In: The Workshop on complex fluids and plasmas, Eindhoven univ. of technology, September, 1995.

18. Meijer E.J., Frenkel D. Colloids dispersed in polymer solutions. A computer simulation study. // J. Chem. Phys., 1994, vol. 100, p. 6873.

19. Bolhuis P.G., Frenkel D. Numerical study of the phase diagram of a mixture of spherical and rodlike colloids. // J. Chem. Phys., 1994, vol. 101, p. 9869.

20. Levin Y., Barbosa M.C., Tamashiro M.H. Liquid state theory of charged colloids. // Europhys. Lett., 1998, vol. 41, No. 2, p. 123-127.

21. Ornstein L.S., Zernike F. Integral equation in liquid state theory. // Proc. Sect. Sci. K. ned. Akad. Wet., 1914, vol. 17, p. 793.

22. Balescu R. Equilibrium Statistical Mechanics. Wiley, 1978.

23. Kirkwood J.G. Theory of Solutions. 1968, Gordon and Breach, New York.

24. Hastings R. On the crystallization of macroionic solutions. // J. Chem. Phys., 1978, vol. 68 , No. 2.

25. Forciniti D., Hall C.K. Structural properties of mixtures of highly asymmetrical electrolytes and uncharged particles using the hypernetted chain approximation. // J. Chem. Phys., 1994, vol. 100, No. 10.

26. Vlachy V. Correlations between macroions in mixtures of charged and uncharged macroparticles. // J. Chem. Phys., 1993, vol. 99, No. 1.

27. Kunz W., Calmettes P., Cartailler T., Turq P. // J. Chem. Phys., 1983, vol. 99, p. 2074.

28. Henderson D., Lozada-Cassou M. // J. Colloid Interface Sci., 1986, vol. 114, p. 180.

29. Wang J., Haymet D.J. Integral equation theory for associating liquids: Dimer and trimer concentrations for model 1-3 electrolytes. // J. Chem. Phys., 1994, vol. 100, No. 5.

30. Satheesan Babu C., Toshiko Ichiye. New integral equation theory for primitive model ionic liquids: From electrolytes to molten salts. // J. Chem. Phys., 1994, vol. 100, No. 12.

31. Vompe A.G., Martynov G.A. The bridge function expansion and self-consistency problem of the Ornstein-Zernike equation solution. // J. Chem. Phys., 1994, vol. 100, No. 7.

32. Hoye J.S., Lomba E., Stell G. Interparticle correlations in colloids. // Mol. Phys., 1992, vol. 75 , p. 1217.

33. Sogami I., Ise N. On the electrostatic interaction in macroionic solutions. // J. Chem. Phys., 1984, vol. 81, No. 12.

34. Scham P., Trigger S. Minimal charge of macroions for crystallization of colloidal suspensions. - In: The Workshop on complex fluids and plasmas, Eindhoven univ. of technology, September, 1995. 


\title{
Статичні кореляції у впорядкованих колоїдних системах
}

\author{
О.І.Герасимов ${ }^{1}$, П.П.Дж.-М.Шрам ${ }^{2}$
}

1 Одеський гідрометеорологічний інститут, 270011 Одеса

2 Технологічний університет, Ейнговен, Нідерланди

Отримано 29 грудня 1998 р.

У рамках гіперланцюжкового наближення інтегрального рівняння Орнштейна-Церніке, використовуючи метод критичних мод, вивчаються макроіон-макроіон кореляційна функція і структурний фактор. На основі недавно отриманих результатів у [1-3] для випадку модельної системи, яка складається з суміші заряджених твердих сфер, досліджено роль деталізації при описі властивостей сполук. Зокрема показано, що додавання незаряджених частинок до моделі розчину макрочастинок і контріонів значно впливає на структурний параметр впорядкування, отриманого в рамках методу критичних мод, забезпечуючи близькість теоретичних і відповідних експериментальних результатів.

Ключові слова: кореляції, структурний фактор, суспензії

PACS: $61.20 . G y, 82.40 .-g$ 